\title{
In-Vitro Comparative Quality Assessment of Different Brands of Doxycycline Hyclate Finished Dosage Forms: Capsule and Tablet in Jimma Town, South Weast Ethiopia
}

\author{
Woldemichael Abraham Leficho ${ }^{1}$, Habtamu Abuye Lambore1, ${ }^{1,}$, Selass Kebede Olbemo ${ }^{1}$, Sultan \\ Suleman Wega ${ }^{2}$ \\ ${ }^{1}$ Department of Pharmacy, College of Medicine and Health Sciences, Wachemo University, Hossana, Ethiopia; \\ ${ }^{2}$ Director of Jimma University Laboratory of Drug Quality, Department of Pharmaceutical Quality Analysis \\ and Regulatory affairs, School of Pharmacy, College of Health Sciences, Jimma University, Jimma, \\ Ethiopia;..... \\ * Correspondence: e-mail-harohabe@gmail.com; Tel.: (+251) 013571974
}

\begin{abstract}
Persistent post market quality evaluation helps produce clear information on the current quality status of the different brands of a given drug and hence introduces biopharmaceutically and therapeutically equivalent list of the products to the prescribers and users of it. This in turn facilitates access to essential medicines by breaking the high cost barrier imposed by few expensive brands of the product. This study was aimed at determining the quality and evaluating equivalence of doxycycline hyclate capsules and tablets in Jimma, Ethiopia. Ten brands of doxycycline hyclate capsules and tablets were tested for physic-chemical parameters. All investigated brands of doxycycline complied with the USP for dosage uniformity, assay of active ingredient and single-point dissolution tests. One brand, D09 failed both hardness and friabilty tests. Comparisons of dissolution profiles applying fit factors confirmed that only brands D04, D06 and D07 had similarity with innovator. Ratio test approaches also showed significant variability existence between test products and comparators. This may question the product's being categorized as a <biowaiver $>$ to mean in-vitro dissolution evaluation suffices its market approval. This work, however, found equivalence problems between 'comparators' and interchangeability issues among doxycycline different brands already distributed in the market.
\end{abstract}

Keywords: Doxycycline; assay; dosage uniformity; hardness, friability, dissolution profile.

\section{Introduction}

The choice of drugs in the management of any disease is an important aspect being considered by healthcare practitioners, patients, government and health insurance companies. [1], [2] As cost is one of the barriers to Essential Medicines, especially in Low Income Countries who have to pay out of pocket for medicines, use of generic medicines solves the problem in part because generic medicines are $20-90 \%$ cheaper than their counterpart innovator medicines.[3] Since the use of generic medicines provide substantial savings to healthcare systems in recent years, many governments and third party payers have advocated utilization of generic medicines as a means of confronting the escalation of healthcare expenditure in general and medicine expenditure in particular, by instigating various policies, initiatives and strategies.[4]

The concern about lowering health care costs has resulted in a tremendous increase in the use of generic drug products. However, providing generic drugs from multiple sources into the healthcare system as a means of reducing healthcare costs is associated with substandard, spurious, falsely labeled, falsified and counterfeit medicines.[5] 
Currently, it is estimated that $10-15 \%$ of the global drugs supplied are counterfeit. The prevalence is higher in developing countries, in Africa and in parts of Asia and Latin America where up to $30-60 \%$ of drugs on the market are counterfeit.[6] Among the medicines, antibiotics account for $28 \%$ of global counterfeit medicines.[7] These problems have resulted in a weak therapeutic efficiency, development of dire resistant strains.[8] There is, therefore, a need to routinely assess the pharmaceutical quality of drugs.

On the other hand, advocating strategy for utilization of generic medicines, as seen from pilot study, was wrongly interpreted by the clients (which is most likely sown and cultivated by drug promoters and intensified through health service givers). As informal market assessment of Jimma town indicated, there was a huge price difference (18.57 times) among the different brands of doxycycline $100 \mathrm{mg}$ capsules and tablets in licensed pharmaceutical retail outlets. Patients, especially from private health institutions, doubt the efficacy, safety and quality of the cheaper products. They were convinced to buy the prescribed brand even he/she has economical constraint to afford. When they were counseled to take an alternative affordable one, their feeling showed unwillingness to adhere to that generic product. Pharmacists were seen acting as seller only instead of discussing the drug issues that the patients have. The both side problems reside on fewer/absence of published studies that informs and assures the patients and the health professionals concerning the performance of different drug brands.

Few studies conducted in Ethiopia on comparative in vitro bioequivalence evaluation of different drug brands reported that: $62.5 \%$ brands of amoxicillin capsules were not interchangeable with the innovator,[9] only one out of five amoxicillin capsule had similar dissolution profile with the innovator and can be considered bioequivalent and interchangeable,[10] and 10 products of co-trimoxazole tablets investigated, the cheaper ones exhibited delayed release during dissolution testing and they released smaller amount of drug compared to the others.[11]

Therefore, it is necessary to compare the existing brands of drug products in order to identify the brands that fit their purpose and can be used interchangeably with the comparator (innovator product). This paper tried to show mainly performances of different brands of doxycycline $100 \mathrm{mg}$ capsules and tablets found in Jimma market. Since doxycycline hyclate is a BCS-I, bio waiver product, that is, in vitro quality evaluation result only suffices to decide on the in-vivo pharmacokinetic properties of the product.[12]

Doxycycline, (chemical structure presented in S1 Supporting information) is a semi-synthetic second-generation tetracycline that came into use in 1967. It is better absorbed after oral administration than first-generation counterparts due to its higher lipid solubility; freely soluble in water and in methanol, sparingly soluble in ethanol (96\%), and BCS class I product for which bio waiver is applied. [12], [13] The drug is chosen for its broad spectrum - high activity against nearly all gram-positive and gram-negative anaerobic and aerobic bacteria, Mycoplasmas, Chlamydiae, Rickettsiae, and some Protozoa.[14]

Doxycycline demonstrates immune-modulating activities that have been used in the treatment of numerous inflammatory conditions mediated by matrix metalloproteinase (MMP-9) and recover inflammatory biomarkers in patients with abdominal aortic aneurysms. It helps to prevent periodontitis and acute coronary syndromes. In recent times, the advancement in the study of tetracycline has developed due to their ability to inhibit matrix metalloproteinase (MMPs) in a variety of cancers such as breast, colorectal, osteosarcoma, melanoma, leukemia and prostate cancers.[15] It has shown favorable effects in trial models of pulmonary fibrosis, emphysema, asthma and acute lung injury.[16] It also provides anti-resorption result by the inhibition of clastic cells and prevent tissue breakdown by the inhibition of mammalian collagenases. It blocks excess of tissue collagenases which is present in periodontitis, consequently leading to enhanced formation of bone and collagen.[17]

Doxycycline is a widely used antibiotics among its class listed in Essential Medicines List of Ethiopia.[18] Typhus, the more common, among the indications in Ethiopia; because the hygiene and sanitation condition is conducive to the vector of the disease-lice, and increases the need for doxycycline hycilte. A study in Kality prison, Addis Ababa, revealed that serological prevalence of 
typhus fever was (26.3\%).[19] In addition to its numerous indications, empirical treatment strategy and non-specific laboratory test (weil-felix in typhus case) has increased the consumption of doxycycline even more from the real demand.[20] This may attract the attention of counterfeiters since the counterfeiting act is focused in highly prescribed or demanded products of a given country or geographical area.[21] Therefore, the present study is mainly aimed at comparison of the different brands of doxycycline $100 \mathrm{mg}$ capsule and tablet with the comparators and in the way it tried to identify whether there was poor quality samples.

\section{Materials and Methods}

\section{Materials}

Doxycycline USP Standard (15008, Lot II) was kindly donated from Cadila Pharmaceuticals Ethiopia. Purified ultra-pure water obtained by water purification system (Thermofischer Scientific, USA, $18.2 \mathrm{M} \Omega . \mathrm{cm}$ at $25^{\circ} \mathrm{C}$ ) which is found in Jimma University Laboratory of Drug Quality (JULaDQ) Jimma, Ethiopia. All other chemicals used in this study were analytical grade and used as received.

\section{Sample collection}

All available ten brands of doxycycline capsules and tablets, each with a label claim of $100 \mathrm{mg}$ were purchased from licensed drug retail outlets in Jimma town, Ethiopia. When arriving at drug outlets, we informed the purpose and asked to buy doxycycline capsule and tablets available for sale which we did not obtained during our previous collection regardless of their price range. During sample collection, all the necessary information about the samples was recorded in a standard report form. Almost all drug retail outlets in Jimma town were visited and only 10 brands were obtained. If a drug outlet had more than one brand, then all the different brands were taken. The experimental part of the work was undertaken at Jimma University drug quality laboratory (JuLaDQ), and the study was performed before product expiration dates. Comparator products selection was based on standard guidelines.[22] Detailed information on doxycycline capsules and tablets included in the study is given in Table 1. Simple codes were given for the products.

\section{Instruments}

HPLC (Agilent 1260 Series, Darmstadt, Germany), Analytical Balance (Mettler Toledo, Greifensee, Switzerland), RC-6D Dissolution Apparatus (Apparatus 1 and Apparatus 2; Tian Jin Optical Instruments, Tianjin, China), UV-Vis Spectrophotometer (Cecil Instruments, Cambridge, United Kingdom), Hardness Tester (Pharma Test, Hainburg, Germany), Friability Tester (Pharma Test), and Water Purification System (Thermo Scientific, Model-7143, Waltham, MA, USA) Thermometer,, Sonicator, Bath UltrasonicElma ${ }^{\circledR}$, Suction Vacuum Pump (Gast Manufacturing Inc.and Standard laboratory glassware were used for the study.

\section{Chemicals and Reagents}

Doxycycline Reference Standard (Assay: 98.99\%, WS/15008, Lot II,) Tetra butylammonium hydrogen sulfate, Tertiary butyl alcohol and Editate disodium were kindly gifted by CADILA Pharmaceuticals Ethiopia. Sodium hydroxide $50 \%$, Potassium phosphate monobasic $\left(\mathrm{KH}_{2} \mathrm{PO}_{4}\right)$ 99-101\%, Ultra-pure water, Hydrochloric acid (36\%, $11.65 \mathrm{M}$, density $1.18 \mathrm{~g} / \mathrm{L})$, Filter papers (Pore Size, $0.45 \mu \mathrm{m}$ ) were supplied by Jimma University Laboratory of Drug Quality (JuLaDQ).

\section{Test methods}

The quality control laboratory tests were performed in JuLaDQ. The laboratory tests were carried out according to the general and individual monographs (for tablet and capsule samples) specified in United States Pharmacopoeia. Instrument performance and system suitability tests were successfully performed for the analytical instruments and HPLC methods, respectively. Quality testing parameters based upon which the products were evaluated as described in conventional monographs were (i) physical tests- uniformity of weight, friability, tablet hardness (ii) chemical tests for content of active ingredients and identity and (iii) in vitro dissolution tests. 


\section{Physical tests}

As comparison with the authentic drug product is always preferred, the first step in evaluating quality of medicine is checking the packaging/labeling and dosage form of the sampled medicine. It aids in identifying suspicious products. Before we begun lab test, both dosage form samples were undergone visual inspection. Tablets must be able to withstand the rigors of handling and transportation experienced in the manufacturing plant, in the drug distribution system, and in the field at the hands of the end users (patients/consumers). Doxycycline hycilate tablets were tested for hardness as per its monograph. Tablets are subjected to tumbling which is consistent with the level and time encountered during manufacture, dedusting, coating, handling, transport and packaging and of course with the patient. The test measures the way tablets hold up under tumbling conditions. The stress of tumbling and resistance of chipping and abrasion is measured by the weight loss of the tablets, after testing and the parameter applied to test this phenomenon is friability. Doxycycline hycilate tablets as well challenged for friability test according to the general pharmacopieal specification. Uniformity of dosage unit is the degree of uniformity in the amount of the drug substance among dosage units. It can be demonstrated by either through content Uniformity (by assay test) or weight variation. Doxycycline hycilate capsules and tablets were evaluated for weight variation.

\section{Chemical tests}

Assay test is a critical quality attribute required to confirm that the labelled amount of drug is available in the given dosage form. The study samples were assayed for their content of Doxycycline hyclate according to their individual monographs described in the USP 38 . The samples were tested for identity via chromatographic peak retention times comparison of RS and test samples.

\section{In-vitro dissolution tests}

The dissolution test is intended to measure the time required for a given drug in an oral solid dosage form to go into solution under specified set of conditions. It is a key analytical test used for: (a) formulation and optimization decisions: during product development, for products where dissolution performance is a critical quality attribute, both the product formulation and the manufacturing process are optimized based on achieving specific dissolution targets. (b) equivalence decisions: during generic product development, and also when implementing post-approval process or formulation changes, similarity of in vitro dissolution profiles between the reference product and its generic or modified version are one of the key requirements for regulatory approval decisions. (c) Product compliance and release decisions: during routine manufacturing, dissolution outcomes are very often one of the criteria used to make product release decisions.[23], [24] These and other vital roles make the test a vitro fundamental analytical quality control method.

\section{Dissolution profile comparison}

Dissolution profile is graphical representation (in terms of concentration against time) of complete release of API from a dosage form in an appropriate selected dissolution medium. It reflects API release pattern under the selected condition sets. Its evaluation on solid dosage forms provides better characterization of the dissolution properties of that product.[25]

Dissolution profile comparison helps to assure the similarity in the product performance and green signals to bioequivalence. Most importantly, it can allow to make appropriate necessary change in test formulation to achieve the same profile of the comparator product/brand leader provided that dissolution profile of particular product of the comparator product/brand leader is known.[26] So, to compare the dissolution profiles test samples and also attempted to identify the nature of API release, we have employed some approaches like ANOVA approach, model-independent approach-difference factor $\left(f_{1}\right)$ and similarity factor $\left(f_{2}\right)$ and model-dependent approach-Weibull release model).

\section{Statistical analysis}

The dissolution data was subjected to one-way ANOVA statistical analysis at $95 \%$ CI followed by post hoc test and two tailed t-test. The dissolution data for $100 \mathrm{mg}$ doxycycline capsule and 
tablet brands were compared to their respective innovator brands and differences were considered significant when $P<0.5$.

\section{Model-independent approaches}

Model-independent approaches promote direct comparison of the dissolution data, and the objective is essentially to translate either the profile or profile differences into a single value. Consequently the results do not depend on the selection of the specific parameter for fitting data but on the chosen sampling time $t_{i}(i=1, \ldots, n)$ in the calculation. Model-independent approaches include ratio tests, dissolution efficiency and fit factors.

\section{Fit factors}

Fit factor uses a difference factor $\left(f_{1}\right)$ and a similarity factor $\left(f_{2}\right)$ was proposed to compare dissolution profiles.[26] The difference factor $\left(f_{1}\right)$ calculates the percent difference between the two curves at each time point and is a measure of the relative error between the two curves.

$$
f 1=\left\{\frac{\sum_{i=1}^{n}|R t-T t|}{\sum_{i=1}^{n} R t}\right\} x 100
$$

Where $n$ is the number of time points, $R_{t}$ is the dissolution value of the reference formulation at time $t$ and $T_{t}$ is the dissolution value of the test formulation at time $t$.

The similarity factor $\left(f_{2}\right)$ is a logarithmic reciprocal square root transformation of the sum of squared error and is a measurement of the similarity in the percent $(\%)$ dissolution between the curves.

$$
f 2=50 \log \left\{\frac{1}{\sqrt{1+\frac{1}{n} \sum(R t-T t)^{2}}}\right\} \times 100
$$

The values of $f_{1}$ ranges from 0 to 15 while $f_{2}$ ranges from 50 to 100 . A test product is similar and hence equivalent to a reference product if $f_{1} \leq 15$ and $f_{2} \geq 50$. Also, two products are dissimilar and hence non-equivalent when $f_{1}>15$ and $f_{2}<50$.[26], [27]

\section{Dissolution Efficiency (DE)}

It is defined as the area under the dissolution curve up to a certain time $(t)$, expressed as a percentage of the area of the rectangle described by $100 \%$ dissolution in the same time point.[28]-[30] It is obtained using the following equation:

$$
\mathrm{DE}=\frac{\int_{t 1}^{t 2} y \mathrm{dt}}{y 100(t 2-t 1)} \times 100
$$

Where, $y$ is the percentage of dissolved product.

The integral of the numerator which is the area under the curve was calculated using the trapezoidal method.[31]

$$
\mathrm{AUC}=\sum_{i=1}^{n} \frac{\left(t_{1}-t_{i-1}\right)\left(y_{i-1}-y_{i}\right)}{2}
$$

Where $t_{i}$ is the $i^{\text {th }}$ time point, $\mathrm{y}_{\mathrm{i}}$ is the $\%$ of dissolved product at time $\mathrm{t}_{\mathrm{i}}$.

\section{Ratio-test approach}

Ratio tests are performed as ratios of percent drug dissolved, DE and mean dissolution times of the reference formulation with those of a test formulation at the same sampling time.[28] The most common ratio test is performed by comparison of two mean dissolution times (MDTs), which are calculated by: 


$$
\mathrm{MDT}=\frac{\sum_{\mathrm{i}}^{\mathrm{n}} \overline{\mathrm{i}} \Delta \mathrm{Mi}}{\sum_{\mathrm{i}}^{\mathrm{n}} \Delta \mathrm{Mi}}
$$

Where $i$ is the sample number, $n$ is the number of dissolution sample times, $\bar{t}=\frac{(\mathrm{ti}-1+\mathrm{ti})}{2}$ is the time at midpoint between $t_{i-1}$ and $t_{i}$, and $\Delta M i$ is the additional amount of drug dissolved between $t_{i-1}$ and $t_{i}$. More precisely, the equation will be rearranged to:

$$
M D T=\frac{\sum t_{I} \Delta M_{i}}{M_{\infty}}
$$

Where $t_{i}$ is an intermediate time of the intervals of sampling time, $\Delta \mathrm{M}_{\mathrm{i}}$ is the amount of API dissolved in every interval of $t$ and $M_{\infty}$ is the maximum of API dissolved.

The dissolution efficiency and the MDT of each of the products were calculated using KineticDS3 software. The dissolution profiles of the products along with their respective sampling points were fed individually to the software.

\section{Model-Dependent Methods}

Model-dependent methods explore the mathematical equations governing the liberation profile as a function of certain parameters related to the pharmaceutical dosage form. These models allow an easy quantitative interpretation of data. Different mathematical models have been proposed to analyze the dissolution profiles through which the mechanism of drug release can be determined. The mathematical models of a dissolution profile can be deduced by a theoretical analysis of the process, such as zero-order kinetics, first-order kinetics, Hixson-Crowell and Weibull models. In this work, doxycycline drug release kinetics was analyzed by them considering the amounts of drug released up to $90 \min [32]-[34]$

\section{A. Zero order model}

Dissolution of the drug from pharmaceutical dosage forms that do not disaggregate and release the drug slowly can be represented by the following equation:

$$
Q_{t}=Q_{0}-k_{0} t
$$

Where, $Q_{0}$ is the initial amount of drug in the pharmaceutical dosage form $Q_{t}$ is the amount of drug in the pharmaceutical dosage form at time $\mathrm{t}$ and $\mathrm{K}$ is proportionality constant.

The pharmaceutical dosage forms following this profiles release the same amount of drug by unit of time and it is the ideal method of drug release in order to achieve a pharmacological prolonged action.

\section{B. First order model}

The application of this model to drug dissolution studies was first proposed by Gibaldi and Feldman.[35] The release of the drug which followed first order kinetics can be expressed by the equation:

$$
\log Q_{t}=\log Q_{0}-\frac{k t}{2.303}
$$

Where, $\mathrm{Q}_{0}$ is the initial concentration of drug, $\mathrm{k}$ is the first order rate constant, and $\mathrm{t}$ is the time. The data obtained are plotted as log cumulative percentage of drug remaining vs. time which would yield a straight line with a slope of $-\mathrm{k} / 2.303$.

\section{Hixson-Crowell model}

Drug powder that having uniformed size particles, Hixson and Crowell derived the equation which expresses rate of dissolution based on cube root of weight of particles and the radius of particle is not assumed to be constant. 
This is expressed by the equation,

$$
M_{0}^{\frac{1}{3}}-M_{t}^{\frac{1}{3}}=\kappa t
$$

Where, M0 is the initial amount of drug in the pharmaceutical dosage form, Mt is remaining amount of drug in the pharmaceutical dosage form at time ' $\mathrm{t}$ ' and $\mathrm{K}$ is proportionality constant incorporating the surface- volume relation. The equation describes the release from systems where there is a change in surface area and diameter of particles or tablets.[35] To study the release kinetics, data obtained from in vitro drug release studies were plotted as cube root of drug percentage remaining in matrix versus time.

\section{Weibull model}

This model has been described for different dissolution processes as the equation.

$$
M=M_{0}\left\{1-e^{\frac{(t-T)^{\beta}}{\alpha}}\right\}
$$

Where, $M$ is the amount of drug dissolved as a function of time $t$. Mo is total amount of drug being released. $T$ accounts for the lag time measured as a result of the dissolution process. Parameter $\alpha$ denotes a scale parameter that describes the time dependence, while $\beta$ describes the shape of the dissolution curve progression.

The model is more useful for comparing the release profiles of matrix type drug delivery.

\section{Results and Discussion}

A total of ten doxycycline capsules and tablets samples were collected between July and June 2016 from Jimma Town licensed pharmaceutical retail outlets (Table 1). Of these, 3 samples were tablets and 7 were capsules. The products were manufactured locally and imported from Asia and Europe.

Table 1. Profile of doxycycline hyclate $100 \mathrm{mg}$ capsule and tablet brands marketed in Jimma,

\begin{tabular}{|c|c|c|c|c|c|c|}
\hline $\begin{array}{c}\text { Sample } \\
\text { Code }\end{array}$ & $\begin{array}{c}\text { Brands } \\
\text { Name }\end{array}$ & Supplier & $\begin{array}{c}\text { Batch } \\
\text { number }\end{array}$ & Mfg date & $\begin{array}{c}\text { Expiry } \\
\text { date }\end{array}$ & Origin \\
\hline D01 & Teradoxin & Huons Co., Ltd & CBC 503 & $09 / 2015$ & $09 / 2018$ & S. Korea \\
\hline D02 & Medomycin & Medochemieltd., Limassol-Cyprus Europe. & APL028 & $11 / 2013$ & $11 / 2017$ & Cyprus \\
\hline D03 & Doxylagap & Labatec-Pharma SA Meyrin for Lagap SA Vezia/Switzerand & 2708 & $11 / 2014$ & $11 / 2017$ & Switzerand \\
\hline D04 & Doxyleb & Leben LaboratoriesPVt. LTD & C-106 & $04 / 2016$ & $03 / 2018$ & India \\
\hline D05 & Epadoxine & East African Pharmaceuticals & F1202 & * & $11 / 2018$ & Ethiopia \\
\hline D06 & Doxycap & Addis Pharmaceuticals Factory & 20644 & * & $07 / 2020$ & Ethiopia \\
\hline D07 & Doxycad & Cadila Pharmaceuticals Ltd & 014013BX58 & $05 / 2016$ & $04 / 2018$ & Ethiopia \\
\hline D08 & Doxy denk & Artesan Pharma GmbH \& Co KG Prinzregentenstr. Germany & 2957 & $09 / 2015$ & $08 / 2018$ & Germany \\
\hline D09 & Miraclin & Laboratorio Farmacologico Milanese s. r. I-Italy & 010216 & $02 / 2016$ & $02 / 2019$ & Italy \\
\hline D10 & Remycin & Remedica, Limassol-Cyprus Europe & 68832 & $06 / 2016$ & $06 / 2021$ & Cyprus \\
\hline
\end{tabular}
Ethiopia

D01-D07 - are capsule dosage forms, D08-D10 - are tablet dosage forms, ${ }^{*}$ - Not found, Mfg-manufacturing

The results of the different quality control tests of the samples are presented in Table 2 and 3 and are detailed below.

Table 2. Pharmacopoeial quality test results for doxycycline hycilate $100 \mathrm{mg}$ capsules.

\begin{tabular}{l|l|l|l|l}
\hline $\begin{array}{l}\text { Sample } \\
\text { Code }\end{array}$ & Assay $(+S D), n=20$ & $\begin{array}{l}\text { Compliance with } \\
\text { USP Assay Test }\end{array}$ & Average Net Weight $(g m), n=10(+S D)$ & $\begin{array}{l}\text { Compliance with Int. Ph } \\
\text { weight variation test }\end{array}$ \\
\hline
\end{tabular}




\begin{tabular}{|c|c|c|c|c|}
\hline D01 & $97.70(0.06)$ & Passed & $362.68(2.68)$ & Passed \\
\hline D02 & $116.0(0.16)$ & Passed & $330.86(3.98)$ & Passed \\
\hline D03 & $99.40(0.13$ & Passed & $283.91(3.63)$ & Passed \\
\hline D04 & $94.70(0.13)$ & Passed & 221.08 & Passed \\
\hline D05 & $93.40(0.23)$ & Passed & $127.22(1.74)$ & Passed \\
\hline D06 & $94.60(0.05)$ & Passed & $222.54(7.03)$ & Passed \\
\hline D07 & $99.30(0.09)$ & Passed & $140.32(9.25)$ & Passed \\
\hline
\end{tabular}

Table 3. Pharmacopoeial quality test results for doxycycline hycilate $100 \mathrm{mg}$ tablets.

\begin{tabular}{|c|c|c|c|c|c|c|c|c|}
\hline $\begin{array}{c}\text { Sample } \\
\text { Code }\end{array}$ & $\begin{array}{c}\text { Assay } \\
(+S D), n=20\end{array}$ & $\begin{array}{c}\text { Compliance } \\
\text { with USP } \\
\text { Assay Test }\end{array}$ & $\begin{array}{r}\text { Average } \\
\text { Weight } \\
(g m), n=20(+S D)\end{array}$ & $\begin{array}{c}\text { Compliance } \\
\text { with USP } \\
\text { weight } \\
\text { variation } \\
\text { test } \\
\end{array}$ & $\begin{array}{l}\text { Friability } \\
(\%), n=10\end{array}$ & $\begin{array}{c}\text { Compliance } \\
\text { with USP } \\
\text { Friability } \\
\text { Test }\end{array}$ & $\begin{array}{c}\text { Average } \\
\text { Hardness in } \\
\text { Newton }\end{array}$ & $\begin{array}{c}\text { Compliance } \\
\text { with USP } \\
\text { Hardness } \\
\text { Test }\end{array}$ \\
\hline D08 & $119.6(0.07)$ & Passed & $259.07(1.85)$ & Passed & 0.17 & Passed & $54.00(4.06)$ & Passed \\
\hline D09 & $102.0(0.21)$ & Passed & $264.97(1.68)$ & Passed & 13.78 & Failed & $31.83(2.07)$ & Failed \\
\hline D10 & $92.60(1.82)$ & Passed & $211.98(2.13)$ & Passed & 0.01 & Passed & $83.64(6.49)$ & Passed \\
\hline
\end{tabular}

Visual Inspection. Neither of the dosage forms nor the dosage units of any of the samples inspected were found defective. No defect of packaging and labeling was encountered.

Identification. All samples had the intended active ingredient as demonstrated by comparing the retention time of the standard and of the samples.

Assay. The assay values for doxycycline hyclate capsules ranged from 93.40-116.00\%lc (mean: 99.3\%), while that of tablets ranged from 92.60 to $119.62 \%$ lc (mean: $104.74 \%$ ). According to USP 38, doxycycline hyclate capsules and tablets contain not less than 90.0 percent and not more than 120.0 percent of the labeled amount of doxycycline API. Although the study samples comply the standard for assay, 4 products have assay values more than 5\% below the label claim (lc) and 2 products have assay values more than $15 \%$ of the target values of the manufacturers.

To identify whether there is a significant variation in the assay values of the tested products, the assay values of the products was checked by single factor ANOVA. The test indicated that there is a significant variation between the amounts of API contents of the different brands.

Dosage uniformity. Dosage uniformity is measured to ensure a constant dose of drug between individual dosage forms. All doxycycline hyclate capsules (Table 2) and tablet (Table 3) samples were in line with pharmacopoeial acceptance criteria for uniformity of weight test. The average weight of net weight of capsules ranges from $127.22 \mathrm{mg}$ to $362.68 \mathrm{mg}$. The expected strength of doxycycline is $100 \mathrm{mg}$. The variation in average net weights of the capsules studied reveals that different manufacturers use different and/or same kind(s) of excipients in different proportions in their products. The comparator product (D03) has about $183.91 \mathrm{mg}$ various excipients in it.

The standard deviation which is a measure of variability or dispersion around the mean weight of the twenty capsules sampled was lowest for D05 $( \pm 1.74)$ and highest for D07 $( \pm 9.25)$ whereas the mean weight of the twenty tablets sampled was lowest for D09 $( \pm 1.54)$ and highest for D10 $( \pm 2.13)$. Thus, tablets (D09) had the best uniformity of weight variation while capsules (brand D07) had the highest dispersion/least clustering of samples weight around the mean weight and hence the least uniform brand. The highest dispersion from the mean for the tablet brand D10 was almost equal to the lowest variation from the mean for the capsule brand D01. However, the percent deviation of all samples were less than the upper acceptance limits and 90\% of the tested products were more persistently uniform than the specifications of the European Pharmacopoeia.

Variability in tablet weight could be the result of defective formulation and production processes such as poor weighing of active pharmaceutical ingredients and excipients, poor mixing of ingredients and changes in tablet compression force applied. While in capsule weight variation arise due to formulation practices that do not comply with good manufacturing practices_the vibrations of a capsule filling machine, capsule filling speed, flowability, particle size, the compressibility, air permeability, powder density and environmental conditions; and lead to variation in the content of capsule.[36] 
A variation in tablet/capsule weight could be an indication of a change in the content of API in the drug products. Such formulations expose the patients to pharmacodynamic and pharmacokinetic fluctuations, on consumption.

Friability test. This test was done for doxycycline hyclate tablet samples (Table 3). One product brand Dog was found to be highly friable that cannot hold up under tumbling conditions and failed to meet pharmacopeial specification for friability test. Its percent weight loss for was $13.78 \%$.

Hardness test. Doxycycline hyclate tablet samples were challenged for this test (Table 3). A product which has failed friability test again unable to withstand minimum crushing force applied. However, it has been provided adequate protection by presenting a single strip of 10 tablets (sandwiched in a folded leaflet) in a hard cartoon in a way that withstand the rigors of handling and transportation experienced in the drug distribution and in the field at the hands of the end users.

It is known that the compression force during a tableting process plays an essential role on the overall properties of the products, such as tablet disintegration rate, friability, and hardness. It is therefore concluded that the compression forces used during the manufacturing processes for brands D08 and D10 are likely to be significantly higher than used for brands D09.

Dissolution. Dissolution is the process of extracting the API out of the dosage form solid-state matrix into solution within the gastrointestinal tract. The dissolution of doxycycline hycilate $100 \mathrm{mg}$ capsule brands was rapid with the release of more than $85 \%$ of the labeled amount within 30 min. All capsule samples passed the single point dissolution test specification. Guidelines discourage further dissolution profile evaluation. However, we tried to see dissolution profile evaluation in different methods.

Unfortunately, the dissolution test for the tablets was not within the regulatory limits for definition of immediate release (IR) specifications. Only after 75 minutes more than $85 \%$ of the label claimed content of the drug released. 


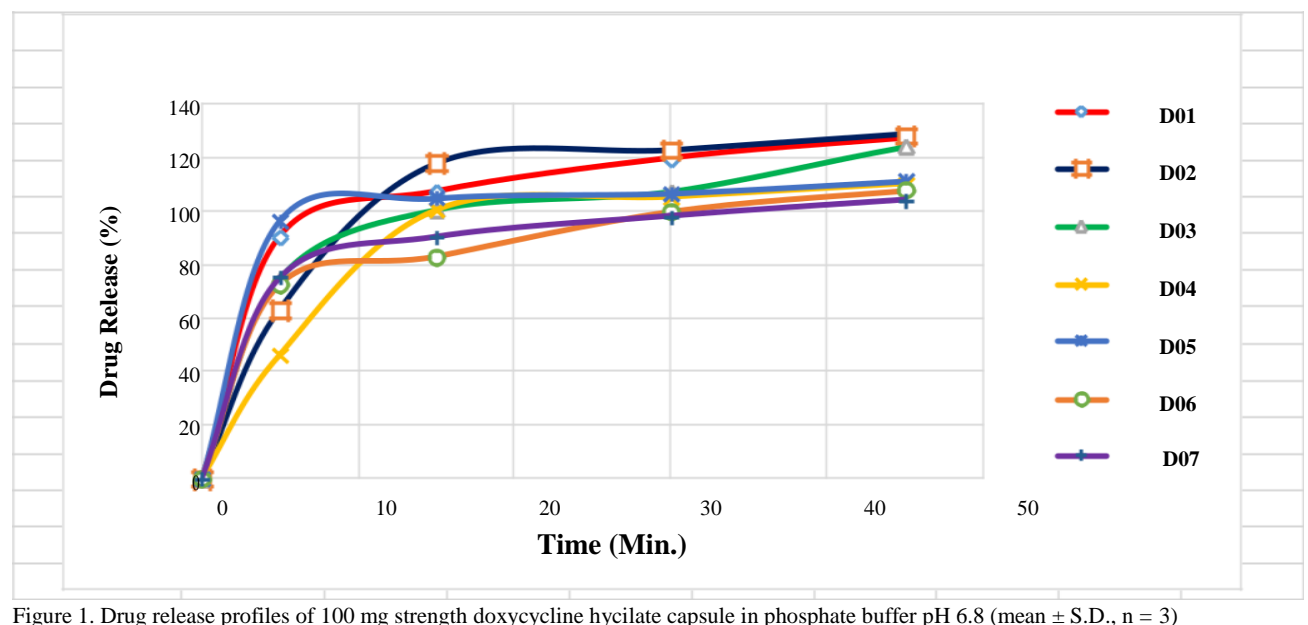

Figure 1. Drug release profiles of $100 \mathrm{mg}$ strength doxycycline hycilate capsule in phosphate buffer $\mathrm{pH} 6.8(\mathrm{mean} \pm$ S.D., $\mathrm{n}=3)$

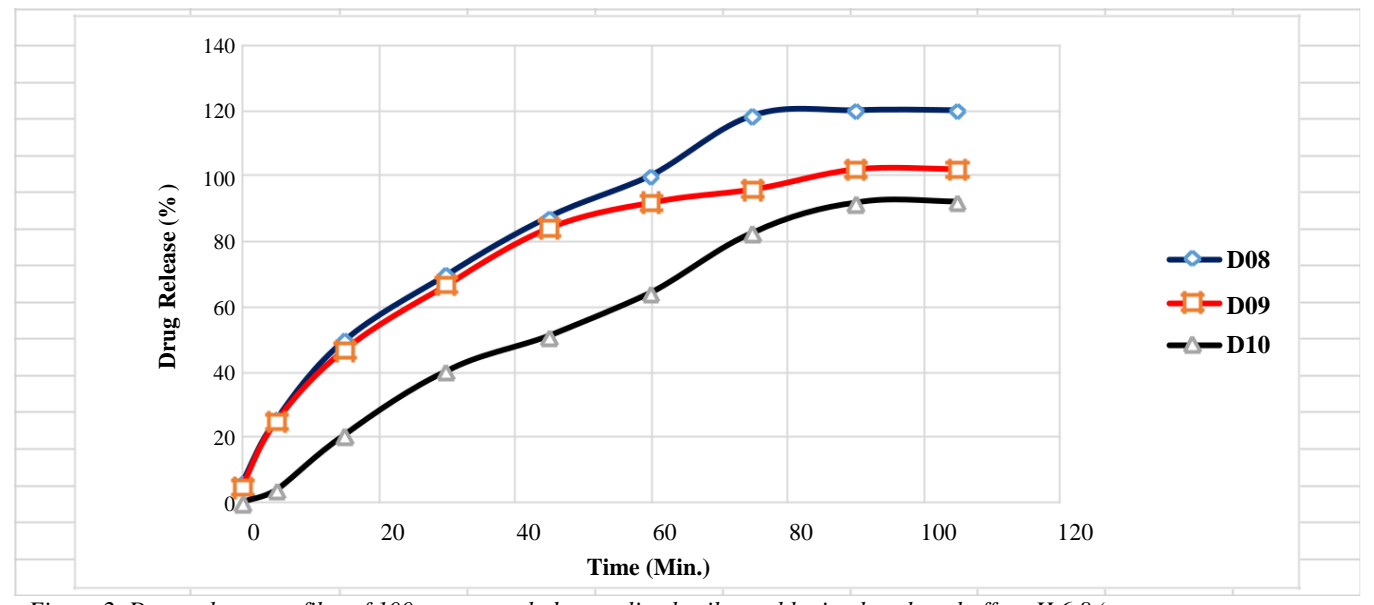

Figure 2. Drug release profiles of $100 \mathrm{mg}$ strength doxycycline hycilate tablet in phosphate buffer $\mathrm{pH} 6.8$ (mean \pm S.D., $n=3$ )

As shown in Figures 1 and 2 drug release at specified points was highly variable. They exhibited different drug release patterns at different time points. Products D03, D04, D06 and D07 within the first 30 minutes the fraction they released reached $\geq 95 \%$ and fulfilled the regulatory limits for definition of rapid dissolution. The remaining capsule samples D01, D02 and D05 released $\geq 95 \%$ of their API before the first 15 minutes has been elapsed and showed to possess very rapid dissolution.

\section{Dissolution profile comparison}

To check whether there is a difference in release profile of brands, one way ANOVA, single factor statistical analysis at 95\% CI was used. The SPSS result showed that there was no significant difference between the released amounts of drug from different brands. To confirm the absence of significant variation, one way ANOVA post hoc test and two tailed t-test for two independent samples were applied. The result showed that the test products and comparator have no significant difference in their release profiles.

Additionally, in order to demonstrate the equivalence of all doxycycline capsules and tablets brands with the comparator products, other approach were employed.

Fit factor $\left(f_{1}\right.$ and $f_{2}$ factors) was performed for six capsules and two tablets brands using their respective comparators D03 and D08 as reference. The calculated results for D10 tablet brand $f_{2}$ value was $<50$ and $f_{1}$ was $>15$. Brand D09 had $f_{2}$ value was $<50$ and $f_{1}$ was $<15$ which is outside acceptable range specified by the USFDA.[27] The tablet brands were dissimilar with the comparator and with one another; because $f_{2}$ value was $<50$ for all the products. Therefore, the tablets are not interchangeable. While capsules D04, D06, and D07 had $f_{2}$ value was $>50$ and $f_{1}$ was $<$ 
15; and for D01 and D05 the $f_{2}$ value was $<50$ and $f_{1}$ was $<15$. As per fit factors specifications, only brans D04, D06, and D07 were interchangeable and equivalent with D03. The remaining capsule brands unable to fit this category since their $f_{2}$ value was $<50$.

Furthermore, interchangeability and/or equivalence was confirmed with comparing DE. DE up to 90 minutes was calculated from the dissolution profile of all brands of doxycycline. As listed in the Table 4, except D04 all tested capsule doxycycline brands had high DE than comparator. To decide which product is equivalent with comparator, their DEs difference has to be within \pm 10 .[37] Accordingly, all products were equivalent to D03 as difference percent (test product - comparator product) is $<10$. Tested tablet samples had generally low DE compared to capsules. Brand D10's, DE difference exceeded the acceptance limit (difference of 12.2\%). It cannot be considered as interchangeable with the D08.

Table 4. Model-Independent Approaches Results of Doxycycline Hycilate $100 \mathrm{mg}$ Brands

\begin{tabular}{llllllllll}
\hline $\begin{array}{c}\text { Sample } \\
\text { Code }\end{array}$ & $f 2$ & $f 1$ & DE (\%) & $\begin{array}{c}\text { \%DE } \\
\text { Difference }\end{array}$ & MDT & $\begin{array}{c}\text { MDT } \\
\text { Ratio }\end{array}$ & $\begin{array}{c}\text { Mean \% } \\
\text { Release } \\
\text { Ratio }\end{array}$ & $\begin{array}{c}\text { SD \% } \\
\text { Release } \\
\text { Ratio }\end{array}$ & $\begin{array}{c}\text { RSD \% } \\
\text { Release } \\
\text { Ratio }\end{array}$ \\
\hline D01 & 64 & 13 & 93.3 & 2.9 & 3.01 & 0.66 & 1.13 & 0.07 & 5.71 \\
D02 & 40 & 18 & 90.3 & 0.1 & 4.32 & 0.88 & 1.08 & 0.19 & 17.47 \\
D04 & 57 & 7 & 86.3 & 4.1 & 6.19 & 1.04 & 0.94 & 0.14 & 15.27 \\
D05 & 38 & 14 & 94.1 & 3.7 & 2.68 & 0.45 & 1.17 & 0.24 & 20.52 \\
D06 & 57 & 8 & 84.0 & 6.4 & 7.20 & 0.95 & 1.05 & 0.09 & 8.10 \\
D07 & 59 & 6 & 87.1 & 3.3 & 5.81 & 0.83 & 1.07 & 0.08 & 7.39 \\
D09 & 41 & 10 & 69.9 & 2.3 & 3.61 & 1.09 & 0.91 & 0.06 & 1.09 \\
D10 & 29 & 22 & 61.0 & 11.2 & 4.18 & 1.23 & 0.77 & 0.04 & 1.23 \\
\hline
\end{tabular}

DS-Standard deviation, RSD - Relative standard deviation, \%DE for D03-90.4\%, for D08-72.2 MDT for D03- 4.22, for D08-3.33

The mean dissolution time was another test category within the model independent method that was employed in this paper. It is determined from the accumulative curves of dissolved API as function of time. The MDT values of D03, D04, D06 and D07; and D01, D02 and D05 had similar dissolution profiles before $30 \mathrm{~min}$. Their MDT values greatly varied after 30 minutes. Brand D01 and D05 showed significant deficiencies compared with those of brands D02, D03, D04, D06 and D07. The MDT values of tablets throughout the run were variable. On the other hand, except D04, all tested brands had MDT ratio greater than one. This indicates that those brands have higher rate of dissolution than the comparators.

The dissolution profiles corresponding to the comparators and other products were evaluated by fitting experimental data to the model-dependent models - the zero order, the first-order, the Hixson-Crowell, and the Weibull models. The model that gives high correlation coefficient, $r^{2}$ value is considered as the best fit of the release data.[38] In Table 5, among the five models fitted to each dissolution profiles, the Weibull model provided the best adjustment curve for all brands, with the higher correlation coefficients. The best fittings were obtained with brands D01, D05 and D10, with maximum determination coefficients. Beyond this, all investigated brands followed the same release mechanism.

Table 5. Model-Dependent Approaches Results of Doxycycline Hycilate 100 mg Brands When Correlation Coefficients were considered

\begin{tabular}{ccccccccccc}
\hline Models & \multicolumn{10}{c}{ Sample Code } \\
\cline { 2 - 11 } & D01 & D02 & D03 & D04 & D05 & D06 & D07 & D08 & D09 & D10 \\
Zero order & 0.3208 & 0.4282 & 0.4282 & 0.5589 & 0.2899 & 0.5939 & 0.5165 & 0.8702 & 0.8962 & 0.9428 \\
First order & 0.2746 & 0.2808 & 0.2790 & 0.2893 & 0.2734 & 0.2844 & 0.2817 & 0.8044 & 0.2271 & 0.7001 \\
Hixson-Crowell & 0.2894 & 0.3402 & 0.3294 & 0.4088 & 0.2785 & 0.3829 & 0.3559 & 0.6789 & 0.6843 & 0.7281 \\
Weibull & 0.9980 & 0.9946 & 0.9958 & 0.9926 & 0.9985 & 0.9924 & 0.9939 & 0.9807 & 0.9873 & 0.9986 \\
\hline
\end{tabular}




\section{Conclusions}

This section is not mandatory, but can be added to the manuscript if the discussion is unusually long or complex.

Author Contributions: Conceptualization, W.A.; methodology, W.A.; software, W.A and H.A.; validation, S.S., ; formal analysis, H.A.; investigation, S.K.; resources, S.K.; data curation, H.A.; writing-original draft preparation, W.A and H.A.; writing-review and editing, S.K.; visualization, S.S.; supervision, S.S.; project administration, S.S. All authors have read and agreed to the published version of the manuscript.

Funding: This research received no external funding.

Acknowledgments: We greatly thank Jimma University for facilitating and providing Lab, and Cadila Pharmaceuticals (Ethiopia) for the donation of reference standard samples. We also appreciate Tesfaye M., Yemer M., Solomon H., Meseret G. and Tamrat B. for their valuable effort on this work.

Conflicts of Interest: The authors declare no conflict of interest.

\section{References}

[1] B. Godman et al., "European payer initiatives to reduce prescribing costs through use of generics," Generics Biosimilars Initiat. J., vol. 1, no. 1, pp. 22-27, 2012, doi: 10.5639/gabij.2012.0101.007.

[2] Y. L. assali, Mohamed \& Shafie, Asrul \& Al-Haddad, M. \& Tangiisuran, Balamurugan \& Awaisu, Ahmed \& Siow, "A qualitative exploration of perceptions toward pharmaceutical price war among community pharmacists in the state of Penang, Malaysia," J. Clin. Diagnostic Res., vol. 13, no. 1, pp. 4453, 2013, doi: https://doi.org/10.1177/1745790413477648.

[3] A. Cameron, M. Ewen, D. Ross-Degnan, D. Ball, and R. Laing, "Medicine prices, availability, and affordability in 36 developing and middle-income countries: a secondary analysis," Lancet, vol. 373, no. 9659, pp. 240-249, 2009, doi: 10.1016/S0140-6736(08)61762-6.

[4] M. A. Hassali et al., "The experiences of implementing generic medicine policy in eight countries: A review and recommendations for a successful promotion of generic medicine use," Saudi Pharm. J., vol. 22, no. 6, pp. 491-503, 2014, doi: 10.1016/j.jsps.2013.12.017.

[5] A. R. Chandrasekaran, C. Y. Han, A. C. Y. Chung, L. W. Cheang, and L. S. Ping, "Post - market In vitro Equivalency Evaluation of Paracetamol Tablets in Kedah , Malaysia," Int. J. Pharm. Sci. Nanotechnol., vol. 4, no. 2, pp. 1403-1407, 2011.

[6] C. A. Delepierre A, Gayot A, “Update on counterfeit antibiotics worldwide; Public health risks," Trends Pharmacol. Sci., vol. 42, no. 6, pp. 247-55, 2012, doi: 10.1016/j.medmal.2012.04.007.

[7] C. A. Delepierre A, Gayot A, “Update on counterfeit antibiotics worldwide; public health risks.,” Med Mal Infect, vol. 42, no. 6, pp. 247-55, 2012, doi: 10.1016/j.medmal.2012.04.007.

[8] F. M. Eckel, "Hospital pharmacy management.," in Southern hospitals, vol. 46, no. 1, 1978, pp. $21-22$.

[9] L. Kassaye and G. Genete, "Evaluation and comparison of in-vitro dissolution profiles for different brands of amoxicillin capsules," Afr. Health Sci., vol. 13, no. 2, pp. 369-375, 2013, doi: 10.4314/ahs.v13i2.25.

[10] G. S. Hailu, G. B. Gutema, and H. Z. Hishe, “Comparative In vitro Bioequivalence Evaluation of Different Brands of Amoxicillin Capsules Marketed in Tigray , Ethiopia," Int. J. Pharm. Sci. Nanotechnol., vol. 6, no. 1, pp. 1966-71, 2013.

[11] The Pharmaceutics and Compounding Laboratory.UNC Eshelman School of Pharmacy, “Evaluation of Tablets." 2019.

[12] E. Jantratid et al., "Biowaiver Monographs for Immediate Release Solid Oral Dosage Forms: 
Doxycycline Hyclate," J. Pharm. Sci., vol. 99, no. 4, pp. 1639-1653, 2010, doi: 10.1002/jps.

[13] W. JM, “The anti-inflammatory effects of tetracyclines,” Cutis, vol. 75, no. 4 Sup, pp. 6-11, 2005.

[14] D. A. R. and A. M. A. Tariq M. Hantash, Ehab A. Abu-Basha, "Pharmacokinetics and bioavailability of doxycycline in ostriches ( Struthio camelus ) at two different dose rates Pharmacokinetics and Bioequivalence of Doxycycline ( Providox ${ }^{\circledR}$ and Doxyvet 0-50 S ${ }^{\circledR}$ ) Oral Powder Formulations in Chickens," Int. J. Poult. Sci., vol. 7, no. 2, pp. 161-164, 2007, doi: 10.4142/jvs.2006.7.4.327.

[15] J. Sagar, K. Sales, S. Dijk, J. Taanman, A. Seifalian, and M. Winslet, “Does Doxycycline work in synergy with cisplatin and oxaliplatin in colorectal cancer ?," World J. Surg. Oncol., vol. 7, no. 2, pp. 1-8, 2009, doi: 10.1186/1477-7819-7-2.

[16] P. M. Beringer, H. Owens, A. Nguyen, D. Benitez, A. Rao, and D. Z. D. Argenio, “Pharmacokinetics of Doxycycline in Adults with Cystic Fibrosis," Antimicrob. Agents Chemother. p., vol. 56, no. 1, pp. 70-74, 2012, doi: 10.1128/AAC.05710-11.

[17] P. V. A. Mohammadi, Zahed, “On the local applications of antibiotics and antibiotic-based agents in endodontics and dental traumatology," Int. Endod. J., vol. 42, no. 7, pp. 555-67, 2009, doi: 10.1111/j.1365-2591.2009.01564.x.

[18] M. W. Aregay, "Food, Medicine and Health Care Administration and Control Authority Bio equivalence Study Registration Requirements in Ethiopia ( Four Countries Experience ),” 2010.

[19] M. W. mariam, B. Gelaw, and A. Assefa, "Seroprevalence of typhus fever at the Kality Prison, Addis Ababa, Ethiopia," Biomed. Res. Ther., vol. 2, no. 7, pp. 318-323, 2015, doi: 10.7603/s40730-015-0016-y.

[20] FMHACA, Standard treatment guidelines for general hospitals, 3 rd. Addis Ababa, 2014.

[21] R. Cockburn, P. N. Newton, E. K. Agyarko, D. Akunyili, and N. J. White, "The Global Threat of Counterfeit Drugs : Why Industry and Governments Must Communicate the Dangers," PLoS Med., vol. 2, no. 4, pp. 302-308, 2005, doi: 10.1371/journal.pmed.0020100.

[22] World Health Organization, “Guidance on the selection of comparator pharmaceutical products for equivalence assessment of interchangeable multisource (generic) products," Geneva, 2015.

[23] Y. Wang, R. D. Snee, G. Keyvan, and F. J. Muzzio, “Statistical comparison of dissolution profiles,” Drug Dev. Ind. Pharm., pp. 1-12, 2015, doi: 10.3109/03639045.2015.1078349.

[24] O. Anand, L. X. Yu, D. P. Conner, and B. M. Davit, “Dissolution Testing for Generic Drugs : An FDA Perspective," AAPS J., vol. 13, no. 3, pp. 328-335, 2011, doi: 10.1208/s12248-011-9272-y.

[25] Shah VP Tsong Y Sathe P Liu JP., "In vitro dissolution profile comparison--statistics and analysis of the similarity factor, f2.," Pharm Res., vol. 15, no. 6, pp. 889-896, 1998, doi: 10.1023/a:1011976615750.

[26] H. H. Moore, J.W. Flanner, “Mathematical Comparison of Dissolution Profiles,” Pharm. Technol., vol. 20, no. 6, pp. 64-75, 1996.

[27] US Food and Drug Administration. Center for Drug Evaluation and and G. for industry Research, Dissolution Testing of Immediate Release Solid Oral Dosage Forms. 1997.

[28] Khan KA Rhodes CT., "Effect of compaction pressure on the dissolution efficiency of some direct compression systems," Pharm Acta Helv., vol. 47, no. 10, pp. 594-607, 1972.

[29] L. Rajput and D. Road, “COMPARATIVE IN VITRO BIOEQUIVALENCE ANALYSIS OF SOME CIPROFLOXACIN HCl GENERIC TABLETS," Int. J. Pharm. Sci. Reseach, vol. 1, no. 8, pp. 51-57, 2010.

[30] T. S. Oishi, I. Nimmi, and S. M. A. Islam, "Comparative in vitro Bioequivalence Analysis of Some Generic Tablets of Atorvastatin , a BCS Class II Compound," Bangladesh Pharm. J., vol. 14, no. 1, pp. 6166, 2011.

[31] S. M. Ashraful-Islam, I. Dewan, M. Shahriar, and M. S. Bin Sayeed, "Validation and Application of a 
Simple HPLC Method for the Comparative In Vitro Dissolution Study of Some Multisource Ciprofloxacin Tablets," IJPI's J. Anal. Chem., vol. 2, no. 8, pp. 1-12, 2012.

[32] F. Langenbucher, "Linearization of dissolution rate curves by the Weibull distribution," J. Pharm. Pharmacol., vol. 24, no. 12, p. 979-981, Dec. 1972, doi: 10.1111/j.2042-7158.1972.tb08930.x.

[33] M. Gibaldi and Stuart Feldman, “Establishment of sink conditions in dissolution rate determinations. Theoretical considerations and application to nondisintegrating dosage forms.," J. Pharm. Sci., vol. 56, no. 10, pp. 1238-1242, 1967, doi: https://doi.org/10.1002/jps.2600561005.

[34] J. G. Wagner, "Interpretation of Percent Dissolved-Time Plots Derived from In Vitro Testing of Conventional Tablets and Capsules," J. Pharm. Sci., vol. 58, no. 10, pp. 1253-1257, 1969, doi: 10.1002/jps.2600581021.

[35] M. Gibaldi D. Perrier, Statistical methods for population pharmacokinetic modeling. Drugs and the Pharmaceutical Sciences, 2 nd. New York: Inc, New York and Basel, 1983.

[36] V. C. Eva Faulhammer, Johannes Khinast, Marcos Llusa, Simon M. Lawrence, Stefano Biserni, “The Effect of Capsule-Filling Machine Vibrations on Average Fill Weight of Capsules," Res. Cent. Pharm. Eng., vol. 454, pp. 381-387, 2013, doi: 10.1016/j.ijpharm.2013.07.029.

[37] N.H.Anderson M.Bauer N.Boussa R.Khan-Malek P.Munden M.Sardaroc, "An evaluation of fit factors and dissolution efficiency for the comparison of in vitro dissolution profiles," J Pharm Biomed Anal., vol. 17, no. (4-5), pp. 811-822, 1998, doi: 10.1016/s0731-7085(98)00011-9.

[38] S. L. J. Costa P, “Modeling and comparison of dissolution profiles,” Eur J Pharm Sci., vol. 13, no. 2, pp. 123-133, 2001. 